\title{
Robust Output Feedback Model Predictive Control for a Class of Networked Control Systems with Nonlinear Perturbation
}

\author{
Qiuxia Chen, ${ }^{1}$ Ying Liu, ${ }^{2}$ and Haoqi Zhu' \\ ${ }^{1}$ Designing Institute, Hangzhou Hangyang Co., Ltd., Hangzhou 310014, China \\ ${ }^{2}$ Department of Information Engineering, Research Center for Smart Agriculture and Forestry, Zhejiang A\&F University, \\ Linian 311300, China \\ Correspondence should be addressed to Ying Liu; zafuliuying@gmail.com
}

Received 20 June 2014; Revised 27 August 2014; Accepted 29 August 2014

Academic Editor: Yun-Bo Zhao

Copyright (C) 2015 Qiuxia Chen et al. This is an open access article distributed under the Creative Commons Attribution License, which permits unrestricted use, distribution, and reproduction in any medium, provided the original work is properly cited.

\begin{abstract}
This paper is concerned with the design problem of robust dynamic output feedback model predictive controllers for a class of discrete-time systems with time-varying network-induced delays and nonlinear perturbation. The designed controllers achieve on-line suboptimal receding horizon guaranteed cost such that the system can be stabilized for all admissible uncertainties. A novel delay compensation strategy is proposed to eliminate the effects of the time-varying network-induced delays. By using multistep prediction and the receding optimization, the delay-dependent sufficient condition is derived for the existence of delay compensation controllers. By employing the cone complementarity linearization (CCL) idea, a nonlinear minimization problem with linear matrix inequality (LMI) constraints is formulated to design the desired output feedback controllers, and an iterative algorithm involving convex optimization is presented to solve the nonlinear minimization problem. Finally, an example is given to illustrate the feasibility and effectiveness of the proposed results.
\end{abstract}

\section{Introduction}

With the rapid development of digital systems and communication networks, more and more control engineers would like to use a real-time communication channel interfaced to a digital system to exchange information and to complete the control task. Such networked control systems (NCSs) have received increasing attention in recent years because of their many advantages, such as lower cost and more convenience for installation and maintenance. Industrial applications of NCSs include automobiles, vehicle systems, robotic systems, jacking systems for trains, and process control systems [1-3]. However, the streams of data exchange between NCS components are prone to delay, losses, and missynchronization, which degrade the performance and even cause the instability of the systems $[4,5]$. Networkinduced delays typically have negative effects on the NCS's stability and performance. So far, different techniques have been presented to deal with the problem of networkinduced delays, such as the stochastic system approach $[6,7]$, the hybrid system approach $[8,9]$, and the time-delay system approach $[10,11]$. It is noted that, in the aforementioned results, all the proposed controllers are operated in an offline fashion, and the controllers are designed such that the overall closed-loop NCSs can tolerate certain amount of network-induced delays. Another constructive scheme is to use future control sequences to directly eliminate the negative effects of the network-induced delays, and model predictive control (MPC) which can provide future control sequences and operate in an on-line fashion is such a desired algorithm.

Model predictive control is probably one of the most successful modern control technologies during recent years. This is due to several thousand applications in process control because of its many advantages, including ease of computation, good tracking performance, and I/O constraint handling capability. Recently, MPC has received increasing attention in NCSs for its ability to on-line compensate time delays as well as its good tracking performance. Reference [12] proposed a new approach of predictive compensation for simultaneous network-induced delays and packet losses and addressed the codesign of both network and controller. In [13], a model-based networked predictive output tracking 
control scheme is proposed to actively compensate for the random round-trip time delay. Reference [14] proposed a data-driven networked predictive control scheme which consisted of the control prediction generator and network delay compensator for MIMO NCSs with random network delays. However, all the above designed model predictive controllers are state feedback controllers which are quite difficult for implementation when the system states cannot be directly measured for availability. Therefore, it is necessary to design a feedback controller using the measured output. To the best of the authors' knowledge, few results have been reported on the dynamic output feedback MPC for NCSs [15]. Besides, the above approaches are not applicable to the case that there are parameter uncertainties in the model. Since the uncertainties are frequently the sources of instability and performance deterioration, the stability analysis and controller synthesis for NCSs with model uncertainty and external disturbances have been some of the most challenging issues. Up to now, a few robust MPC algorithms have been presented to improve the robustness of the networked control systems $[16,17]$.

Taking the network-induced delay into consideration, the NCSs can be modeled as an uncertain discrete-time system with time-varying delays, and this motivates us to apply the theory of time-delay systems and robust MPC strategy to design the feedback controllers for such NCSs. The main results of this paper will contribute to the development of the delay-dependent dynamic output feedback robust MPC for a class of NCSs with norm-bounded nonlinear perturbation and time-varying communication delays. A sufficient delaydependent condition that guarantees the robust stability of the closed-loop NCS is derived. An optimization problem is also formulated to construct the dynamic output feedback MPC controllers subject to a set of nonlinear matrix inequalities. Based on the linearization idea [18], an iterative algorithm involving convex optimization is proposed to solve the nonlinear matrix inequality system, and the iterative optimization algorithm is guaranteed to be feasible at each time step if it is feasible at the first step. The control inputs applied to the system from the solutions of the MPC optimization problems guarantee an on-line suboptimal receding horizon guaranteed cost. Finally, an example is given to illustrate the effectiveness of the proposed results.

Notation. Throughout the paper, $\mathfrak{R}^{n}$ stands for the set of all real $n$-dimensional vectors and $\Re^{n \times m}$ is the set of all $n \times m$-dimensional matrices. I denotes identity matrix of appropriate dimensions; $\operatorname{diag}\{\cdot\}$ denotes the block diagonal matrix. $\mathbf{G}>0(\mathbf{G} \geq 0, \mathbf{G}<0, \mathbf{G} \leq 0)$ means that $\mathbf{G}$ is a real symmetric positive-definite matrix (positivesemidefinite, negative-definite, and negative-semidefinite). * denotes the symmetric part.

\section{Problem Formulation and Preliminaries}

The detailed assumptions about the NCS studied in this paper are described as follows.
(1) The sensor is clock-driven and has the sampling period $h$, and the controller and the actuator are event-driven.

(2) The sensor-to-controller delay $\tau_{k}^{s c}$ and the controllerto-actuator delay $\tau_{k}^{c a}$ are both uncertain but bounded and can be obtained by best case analysis and worst case analysis. The total time delay $\tau_{k}^{s a}=\tau_{k}^{s c}+\tau_{k}^{c a}$ satisfies $\underline{\tau} \leq \tau_{k}^{s a} \leq \bar{\tau}$, where $\bar{\tau}$ and $\underline{\tau}$ are known positive integers corresponding to maximum and minimum of $\tau_{k}^{s a}$.

(3) Controller computational delay can be absorbed into either $\tau_{k}^{s c}$ or $\tau_{k}^{c a}[19]$.

The uncertain discrete-time system with nonlinear perturbation is described by the following state space model:

$$
\begin{gathered}
\mathbf{x}(k+1)=(\mathbf{A}+\Delta \mathbf{A}(k)) \mathbf{x}(k)+(\mathbf{B}+\Delta \mathbf{B}(k)) \mathbf{u}(k) \\
+\mathbf{f}(\mathbf{x}(k), \mathbf{u}(k)), \\
\mathbf{y}(k)=\mathbf{C} \mathbf{x}(k),
\end{gathered}
$$

where $\mathbf{x}(k) \in \mathfrak{R}^{n}$ is the state, $\mathbf{u}(k) \in \mathfrak{R}^{l}$ is the control input, $\mathbf{y}(k) \in \mathfrak{R}^{v}$ is the measured output, and $\mathbf{A}, \mathbf{B}$, and $\mathbf{C}$ are known real constant matrices of appropriate dimensions. $\Delta \mathbf{A}(k)$ and $\Delta \mathbf{B}(k)$ are unknown matrices representing timevarying parameter uncertainties in the system model. It is assumed that the uncertainties are norm-bounded and can be described as $\left[\begin{array}{ll}\Delta \mathbf{A} & \Delta \mathbf{B}\end{array}\right]=\mathbf{D F}(k)\left[\begin{array}{ll}\mathbf{E}_{1} & \mathbf{E}_{2}\end{array}\right]$, where $\mathbf{F}(k) \in \mathfrak{R}^{i \times j}$ is an unknown matrix satisfying $\mathbf{F}^{T}(k) \mathbf{F}(k) \leq \mu \mathbf{I}$ and $\mu$ is a known positive scalar representing the upper bound of the unknown matrix $\mathbf{F}(k)$, and $\mathbf{D}, \mathbf{E}_{1}$, and $\mathbf{E}_{2}$ are known constant matrices of appropriate dimensions. The nonlinear function f satisfies the following:

$$
\mathbf{f}^{T} \mathbf{f} \leq \alpha^{2}\left[\begin{array}{l}
\mathbf{x}(k) \\
\mathbf{u}(k)
\end{array}\right]^{T}\left[\begin{array}{cc}
\mathbf{H}_{1}^{T} \mathbf{H}_{1} & 0 \\
0 & \mathbf{H}_{2}^{T} \mathbf{H}_{2}
\end{array}\right]\left[\begin{array}{l}
\mathbf{x}(k) \\
\mathbf{u}(k)
\end{array}\right]
$$

where $\mathbf{H}_{1}$ and $\mathbf{H}_{2}$ are known constant matrices and $\alpha>0$ is the bounding parameter on the uncertain function $\mathbf{f}$.

The physical system consisting of sensor and actuator nodes is connected to the controller through a communication medium. For the convenience of system analysis and controller design, the sensor-to-controller delay $\tau_{k}^{s c}$ and the controller-to-actuator delay $\tau_{k}^{c a}$ are lumped up as the feedforward delay $\tau(k) h$. Considering the delay effect, the input of the controller can be given as $\mathbf{y}(k)=\mathbf{y}(k-\tau(k))$, and then the full-order dynamic output feedback controller to be determined has the following form:

$$
\begin{gathered}
\widehat{\mathbf{x}}(k+1)=\mathbf{A}_{c} \widehat{\mathbf{x}}(k)+\mathbf{B}_{c} \mathbf{y}(k-\tau(k)) \\
\mathbf{u}(k)=\mathbf{C}_{c} \widehat{\mathbf{x}}(k),
\end{gathered}
$$

where $\widehat{\mathbf{x}}(k) \in \Re^{\mathbf{n}}$ is the controller state.

Applying controller (3) to the system (1) results in the following networked closed-loop system:

$$
\overline{\mathbf{x}}(k+1)=\widetilde{\mathbf{A}} \overline{\mathbf{x}}(k)+\overline{\mathbf{A}}_{d} \mathbf{x}(k-\tau(k))+\overline{\mathbf{I}}^{T} \mathbf{f}(\mathbf{x}(k), \mathbf{u}(k)),
$$


where

$$
\begin{array}{cc}
\overline{\mathbf{x}}(k)=\left[\begin{array}{l}
\mathbf{x}(k) \\
\widehat{\mathbf{x}}(k)
\end{array}\right], & \overline{\mathbf{A}}=\left[\begin{array}{cc}
\mathbf{A} & \mathbf{B C}_{c} \\
0 & \mathbf{A}_{c}
\end{array}\right], \\
\overline{\mathbf{A}}_{d}=\left[\begin{array}{c}
0 \\
\mathbf{B}_{c} \mathbf{C}
\end{array}\right], \quad \overline{\mathbf{D}}=\left[\begin{array}{c}
\mathbf{D} \\
0
\end{array}\right], \\
\widetilde{\mathbf{A}}=\overline{\mathbf{A}}+\overline{\mathbf{D}} \mathbf{F}(k) \overline{\mathbf{E}}, \\
\overline{\mathbf{E}}=\left[\begin{array}{ll}
\mathbf{E}_{1} & \mathbf{E}_{2} \mathbf{C}_{c}
\end{array}\right], \quad \overline{\mathbf{I}}=\left[\begin{array}{ll}
\mathbf{I} & 0
\end{array}\right] .
\end{array}
$$

In this way, the NCS (1) and (3) with nonlinear perturbation and communication delays is modeled as the uncertain discrete-time system (4) with time-varying state delay, which enables us to apply the theory of time-delay systems and the receding optimization of the MPC to deal with the analysis and design problem of such NCS.

Remark 1. Equation (4) is used to express the mathematical model of the networked control systems when the transmitted data is single packet. For the multiple-packet transmission case, since the arrival time of the sensor messages at the controller or the arrival time of the controller messages at the actuator may be different, especially for the case when the sampling times of the sensors are different, a buffer before the controller and actuator is needed. By employing the buffer technology on the network, model (4) can also be used to express the NCS with multiple-packet transmission.

The objective of this paper is to find a stabilizing dynamic output feedback controller of the form (3) for the uncertain system (1) with time-varying delays by MPC strategy. To this end, we define the following min-max optimization problem, which is considered at each sampling time $k$ :

$$
\begin{gathered}
\min _{\mathbf{u}_{k+i \mid k}, i=0,1, \ldots, m[\mathbf{A}(k+i), \mathbf{B}(k+i)], i \geq 0} J(k), \\
J(k)=\sum_{i=0}^{p} \mathbf{x}_{k+i \mid k}^{T} S_{1} \mathbf{x}_{k+i \mid k}+\sum_{i=0}^{m} \mathbf{u}_{k+i \mid k}^{T} \mathbf{S}_{2} \mathbf{u}_{k+i \mid k},
\end{gathered}
$$

where $m$ is the control horizon, $p$ is the prediction horizon, $\mathbf{S}_{1}>0$ and $\mathbf{S}_{2}>0$ are given weighting matrices, and $\mathbf{x}_{k+i \mid k}$ and $\mathbf{u}_{k+i \mid k}$ denote the predicted variables of the state and the input, respectively, with $\mathbf{x}_{k \mid k}=\mathbf{x}(k), \widehat{\mathbf{x}}_{k \mid k}=\widehat{\mathbf{x}}(k)$, and $\mathbf{x}_{k-i \mid k}=\mathbf{x}(k-i)$ for $i \geq 1$. Besides, we have the terminal constraints $\mathbf{u}_{k+i \mid k}=0$ for $i>m$.

Associated with the closed-loop system (4), the min-max optimization problem (6) becomes of the following form:

$$
\begin{gathered}
\min _{\mathbf{u}_{k+i \mid k, i=0,1, \ldots, m}} \max _{[\mathbf{A}(k+i), \mathbf{B}(k+i)], i \geq 0} J(k), \\
J(k)=\sum_{i=0}^{p} \overline{\mathbf{x}}_{k+i \mid k}^{T} \widetilde{\mathbf{S}} \overline{\mathbf{x}}_{k+i \mid k},
\end{gathered}
$$

where $\widehat{\mathbf{x}}_{k+i \mid k}=0, i>m$, and $\widetilde{\mathbf{S}}=\operatorname{diag}\left\{\mathbf{S}_{1}, \mathbf{C}_{c}^{T} \mathbf{S}_{2} \mathbf{C}_{c}\right\}$.

The future control sequence can be obtained by solving the above optimization problem. In order to eliminate the delay effects, the control input should be $\mathbf{u}(k)=u_{k+\tau_{k}^{s a} / h \mid k}$ but not $u_{k+\tau(k) \mid k}$. By using the multistep prediction and the linear interpolation method, the control sequence $\Pi_{k}=$ $\left\{u_{k+\tau_{k}^{s a} / h \mid k}, u_{k+1+\tau_{k}^{s a} / h \mid k}, \ldots, u_{k+\omega+\tau_{k}^{s a} / h \mid k}\right\}$ can be obtained. In the receding horizon framework, only the first control variable $u_{k+\tau_{k}^{s a} / h \mid k}$ actuates the system in time.

Lemma 2 (see [20]). Let $\widehat{\mathbf{J}}=\widehat{\mathbf{J}}^{T}>0$ and $\widehat{\mathbf{H}}, \widehat{\mathbf{L}}$ be given matrices with appropriate dimensions. Then, it follows that

$$
\widehat{\mathbf{J}}+\widehat{\mathbf{H}} \mathbf{F} \widehat{\mathbf{L}}+\widehat{\mathbf{L}} \mathbf{F} \widehat{\mathbf{H}}<0
$$

holds for any matrix $\mathbf{F}$ satisfying $\mathbf{F}^{T} \mathbf{F} \leq \mu \mathbf{I}$, if and only if there exists a scalar $\varepsilon>0$ such that

$$
\mu \widehat{\mathbf{H}} \widehat{\mathbf{H}}^{T}+\varepsilon \widehat{\mathbf{J}}+\varepsilon^{2} \widehat{\mathbf{L}}^{T} \widehat{\mathbf{L}}<0
$$

\section{Main Results}

In this section, we first derive an upper bound on the worst value of the cost $J(k)$.

Theorem 3. Consider the uncertain system (1) and the cost function $J(k)$. If there exist a controller of the form (3), a scalar $\varepsilon \geq \sqrt{\mu}, \mathbf{P}>0 \in \mathfrak{R}^{2 n \times 2 n}, \mathbf{Q}>0 \in \mathfrak{R}^{n \times n}, \mathbf{R}>0 \in \mathfrak{R}^{n \times n}$, and matrices $\mathbf{M}_{0} \in \mathfrak{R}^{n \times n}, \mathbf{M}_{1} \in \mathfrak{R}^{n \times n}$, such that the following matrix inequality holds

$$
\left[\begin{array}{cccccccc}
\mathbf{M}_{a} & * & * & * & * & * & * & * \\
\mathbf{M}_{b} & \mathbf{M}_{c} & * & * & * & * & * & * \\
0 & 0 & \lambda \mathbf{I} & * & * & * & * & * \\
\overline{\mathbf{A}} & \overline{\mathbf{A}}_{d} & \overline{\mathbf{I}}^{T} & \mathbf{P}^{-1} & * & * & * & * \\
\overline{\mathbf{A}}_{f} & 0 & \mathbf{I} & 0 & \bar{\tau}^{-1} \mathbf{R}^{-1} & * & * & * \\
\widetilde{\mathbf{M}}_{0} & \mathbf{M}_{1} & 0 & 0 & 0 & \bar{\tau}^{-1} \mathbf{R} & * & * \\
0 & 0 & 0 & \overline{\mathbf{D}}^{T} & \mathbf{D}^{T} & 0 & \varepsilon^{-1} \mathbf{I} & * \\
\overline{\mathbf{E}} & 0 & 0 & 0 & 0 & 0 & 0 & \varepsilon^{-1} \mathbf{I}
\end{array}\right]>0
$$

where

$$
\begin{aligned}
& \mathbf{M}_{a}=\mathbf{P}-\widetilde{\mathbf{S}}-\mathbf{M}_{d}-\lambda \alpha^{2} \widetilde{\mathbf{H}}, \\
& \mathbf{M}_{d}=\operatorname{diag}\left\{\mathbf{M}_{0}+\mathbf{M}_{0}^{T}-d_{c} \mathbf{Q}, 0\right\}, \\
& d_{c}=\underline{\tau}-\bar{\tau}-1, \\
& \widetilde{\mathbf{H}}=\operatorname{diag}\left\{\mathbf{H}_{1}^{T} \mathbf{H}_{1}, \mathbf{C}_{c}^{T} \mathbf{H}_{2}^{T} \mathbf{H}_{2} \mathbf{C}_{c}\right\}, \\
& \mathbf{M}_{b}=\left[\begin{array}{ll}
\mathbf{M}_{1}^{T}-\mathbf{M}_{0} 0
\end{array}\right], \\
& \mathbf{M}_{c}=\mathbf{Q}+\mathbf{M}_{1}+\mathbf{M}_{1}^{T}, \\
& \overline{\mathbf{A}}_{f}=\left[\begin{array}{ll}
\mathbf{A}-\mathbf{I} & \mathbf{B} \mathbf{C}_{c}
\end{array}\right], \\
& \widetilde{\mathbf{M}}_{0}=\left[\begin{array}{ll}
\mathbf{M}_{0} & 0
\end{array}\right]
\end{aligned}
$$


then the closed-loop system (4) is asymptotically stable and the cost function $J(k)$ satisfies the following:

$$
\begin{aligned}
\max _{[\mathbf{A}(k+i), \mathbf{B}(k+i)], i \geq 0} J(k)< & \overline{\mathbf{x}}_{k \mid k}^{T} \mathbf{P} \overline{\mathbf{x}}_{k \mid k}+\sum_{\theta=k-\bar{\tau}}^{k-1} \mathbf{x}_{\theta \mid k}^{T} \mathbf{Q} \mathbf{x}_{\theta \mid k} \\
& +\sum_{j=-\bar{\tau}}^{-1} \sum_{\theta=k+j}^{k-1} \eta_{\theta \mid k}^{T} \mathbf{R} \eta_{\theta \mid k} \\
& +\sum_{j=-\bar{\tau}+2}^{-\tau+1} \sum_{\theta=k+j-1}^{k-1} \mathbf{x}_{\theta \mid k}^{T} \mathbf{Q} \mathbf{x}_{\theta \mid k},
\end{aligned}
$$

where $\eta_{\theta \mid k}=\mathbf{x}_{\theta+1 \mid k}-\mathbf{x}_{\theta \mid k}$.

Proof. Choose the following candidate Lyapunov functional:

$$
\bar{V}\left(\overline{\mathbf{x}}_{k+i \mid k}\right)=V_{1}\left(\overline{\mathbf{x}}_{k+i \mid k}\right)+V_{2}\left(\overline{\mathbf{x}}_{k+i \mid k}\right)+V_{3}\left(\overline{\mathbf{x}}_{k+i \mid k}\right),
$$

where

$$
\begin{gathered}
V_{1}\left(\overline{\mathbf{x}}_{k+i \mid k}\right)=\overline{\mathbf{x}}_{k+i \mid k}^{T} \mathbf{P} \overline{\mathbf{x}}_{k+i \mid k}, \\
V_{2}\left(\overline{\mathbf{x}}_{k+i \mid k}\right)=\sum_{\theta=k+i-\tau(k)}^{k+i-1} \mathbf{x}_{\theta \mid k}^{T} \mathbf{Q} \mathbf{x}_{\theta \mid k}, \\
V_{3}\left(\overline{\mathbf{x}}_{k+i \mid k}\right)=\sum_{j=-\bar{\tau}}^{-1} \sum_{\theta=k+i+j}^{k+i-1} \eta_{\theta \mid k}^{T} \mathbf{R} \eta_{\theta \mid k}+\sum_{j=-\bar{\tau}+2}^{-\tau+\frac{\tau}{+}+1} \sum_{\theta+i+j-1}^{k+i-1} \mathbf{x}_{\theta \mid k}^{T} \mathbf{Q} \mathbf{x}_{\theta \mid k}, \\
i=0,1, \ldots, p .
\end{gathered}
$$

Taking the forward difference for the Lyapunov functional $V_{1}\left(\overline{\mathbf{x}}_{k+i \mid k}\right)$, one obtains

$$
\Delta V_{1}\left(\overline{\mathbf{x}}_{k+i \mid k}\right)=\boldsymbol{\psi}^{T}(k)\left\{\Gamma^{T} \mathbf{P} \Gamma+\operatorname{diag}\{-\mathbf{P}, 0,0\}\right\} \psi(k),
$$

where

$$
\begin{aligned}
& \Gamma=\left[\begin{array}{lll}
\widetilde{\mathbf{A}} & \overline{\mathbf{A}}_{d} & \overline{\mathbf{I}}^{T}
\end{array}\right],
\end{aligned}
$$

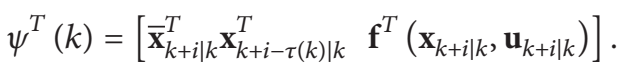

Direct computation gives

$$
\begin{aligned}
\Delta V_{2}\left(\overline{\mathbf{x}}_{k+i \mid k}\right)= & \mathbf{x}_{k+i \mid k}^{T} \mathbf{Q} \mathbf{x}_{k+i \mid k}-\mathbf{x}_{k+i-\tau(k) \mid k}^{T} \mathbf{Q \mathbf { x } _ { k + i - \tau ( k ) | k }} \\
& +\sum_{\theta=k+i+1-\tau(k+1)}^{k+i-1} \mathbf{x}_{\theta \mid k}^{T} \mathbf{Q} \mathbf{x}_{\theta \mid k} \\
& -\sum_{\theta=k+i+1-\tau(k)}^{k+i-1} \mathbf{x}_{\theta \mid k}^{T} \mathbf{Q} \mathbf{x}_{\theta \mid k} .
\end{aligned}
$$

Note that

$$
\begin{aligned}
& \sum_{\theta=k+i+1-\tau(k+1)}^{k+i-1} \mathbf{x}_{\theta \mid k}^{T} \mathbf{Q} \mathbf{x}_{\theta \mid k} \\
& =\sum_{\theta=k+i+1-\underline{\tau}}^{k+i-1} \mathbf{x}_{\theta \mid k}^{T} \mathbf{Q} \mathbf{x}_{\theta \mid k}+\sum_{\theta=k+i+1-\tau(k+1)}^{k+i-\underline{\tau}} \mathbf{x}_{\theta \mid k}^{T} \mathbf{Q} \mathbf{x}_{\theta \mid k} \\
& \leq \sum_{\theta=k+i+1-\tau(k)}^{k+i-1} \mathbf{x}_{\theta \mid k}^{T} \mathbf{Q} \mathbf{x}_{\theta \mid k}+\sum_{\theta=k+i+1-\bar{\tau}}^{k+i-\underline{\tau}} \mathbf{x}_{\theta \mid k}^{T} \mathbf{Q} \mathbf{x}_{\theta \mid k} .
\end{aligned}
$$

Hence, we have

$$
\begin{aligned}
\Delta V_{2}\left(\overline{\mathbf{x}}_{k+i \mid k}\right) \leq & \mathbf{x}_{k+i \mid k}^{T} \mathbf{Q} \mathbf{x}_{k+i \mid k}-\mathbf{x}_{k+i-\tau(k) \mid k}^{T} \mathbf{Q} \mathbf{x}_{k+i-\tau(k) \mid k} \\
& +\sum_{\theta=k+i+1-\bar{\tau}}^{k+i-\underline{\tau}} \mathbf{x}_{\theta \mid k}^{T} \mathbf{Q} \mathbf{x}_{\theta \mid k} .
\end{aligned}
$$

$\Delta \mathbf{V}_{3}\left(\overline{\mathbf{x}}_{k+i \mid k}\right)$ is computed as follows:

$$
\Delta \mathbf{V}_{3}\left(\overline{\mathbf{x}}_{k+i \mid k}\right)=\bar{\tau} \eta_{k+i \mid k}^{T} \mathbf{R} \eta_{k+i \mid k}+(\bar{\tau}-\underline{\tau}) \mathbf{x}_{k+i \mid k}^{T} \mathbf{Q} \mathbf{x}_{k+i \mid k}
$$

$$
-\sum_{\theta=k+i-\bar{\tau}}^{k+i-1} \eta_{\theta \mid k}^{T} \mathbf{R} \eta_{\theta \mid k}-\sum_{\theta=k+i+1-\bar{\tau}}^{k+i-\underline{\tau}} \mathbf{x}_{\theta \mid k}^{T} \mathbf{Q} \mathbf{x}_{\theta \mid k} .
$$

By the well-known inequality $-2 \mathbf{a}^{T} \mathbf{b} \leq \mathbf{a}^{T} \mathbf{R a}+\mathbf{b}^{T} \mathbf{R}^{-1} \mathbf{b}$, we obtain

$$
-\sum_{\theta=k+i-\bar{\tau}}^{k+i-1} \eta_{\theta \mid k}^{T} \mathbf{R} \eta_{\theta \mid k} \leq \psi^{T}(k) \Delta_{1} \psi(k),
$$

where $\Delta_{1}=2\left[\begin{array}{lll}\widetilde{\mathbf{I}} & -\mathbf{I} & 0\end{array}\right]^{T} \widetilde{\mathbf{Y}}+\widetilde{\mathbf{Y}}^{T} \bar{\tau} \mathbf{R}^{-1} \widetilde{\mathbf{Y}}, \widetilde{\mathbf{Y}}=\left[\begin{array}{lll}\widetilde{\mathbf{M}}_{0} & \mathbf{M}_{1} & 0\end{array}\right]$. Combining (15)-(21) yields

$$
\Delta \bar{V}\left(\overline{\mathbf{x}}_{k+i \mid k}\right) \leq \psi^{T}(k) \Pi_{1} \psi(k)-\overline{\mathbf{x}}_{k+i \mid k}^{T} \widetilde{\mathbf{S}} \overline{\mathbf{x}}_{k+i \mid k},
$$

where

$$
\begin{aligned}
& \widetilde{\mathbf{A}}_{f}=\overline{\mathbf{A}}_{f}+\mathbf{D F}(k) \overline{\mathbf{E}}, \\
& \Pi_{1}=\Delta_{1}+\Delta_{2}+\Delta_{3},
\end{aligned}
$$

$$
\begin{gathered}
\Delta_{2}=\left[\begin{array}{ccc}
-\mathbf{P}+\widetilde{\mathbf{S}}+\mathbf{M}_{d} & \mathbf{M}_{b}^{T} & 0 \\
\mathbf{M}_{b} & -\mathbf{M}_{c} & 0 \\
0 & 0 & 0
\end{array}\right], \\
\Delta_{3}=\Gamma^{\mathrm{T}} \mathbf{P} \Gamma+\left[\begin{array}{lll}
\widetilde{\mathbf{A}}_{f} & 0 & \mathbf{I}
\end{array}\right]^{T} \bar{\tau} \mathbf{R}\left[\begin{array}{lll}
\widetilde{\mathbf{A}}_{f} & 0 & \mathbf{I}
\end{array}\right],
\end{gathered}
$$

and $\psi^{T}(k) \Pi_{1} \psi(k)<0$ implies that $\Delta \bar{V}\left(\overline{\mathbf{x}}_{k+i \mid k}\right)<-\overline{\mathbf{x}}_{k+i \mid k}^{T} \widetilde{\mathbf{s}} \overline{\mathbf{x}}_{k+i \mid k}$ $\leq 0$; that is, $\bar{V}\left(\overline{\mathbf{x}}_{k+i \mid k}\right)$ is degenerated. Therefore, the closedloop system (4) is asymptotically stable. Besides, for any integer $K>0$, we have

$$
\begin{aligned}
\sum_{i=0}^{K} \overline{\mathbf{x}}_{k+i \mid k}^{T} \widetilde{\mathbf{S}}_{k+i \mid k} & \leq-\sum_{i=0}^{K} \Delta \bar{V}\left(\overline{\mathbf{x}}_{k+i \mid k}\right) \\
& =-\bar{V}\left(\overline{\mathbf{x}}_{k+K+1 \mid k}\right)+\bar{V}\left(\overline{\mathbf{x}}_{k \mid k}\right) \\
& <\bar{V}\left(\overline{\mathbf{x}}_{k \mid k}\right) .
\end{aligned}
$$


To guarantee the existence of the upper bound on the robust performance index $J(k)$, we must have $\mathbf{x}_{k+K \mid k}=0, K \geq p$; hence, $\bar{V}\left(\overline{\mathbf{x}}_{k+K+1 \mid k}\right)=0, K \geq p$. Let $K \rightarrow p$, and then $\max _{[\mathbf{A}(k+i), \mathbf{B}(k+i)], i \geq 0} J(k) \leq \bar{V}\left(\overline{\mathbf{x}}_{k \mid k}\right)$; we get (12).

By Schur complement, (2) is equivalent to the following matrix inequality:

$$
\psi^{T}(k) \Pi_{2} \psi(k) \geq 0
$$

where $\Pi_{2}=\operatorname{diag}\left\{\alpha^{2} \widetilde{\mathbf{H}}, 0,-\mathbf{I}\right\}$.

By $S$-procedure, $\psi^{T}(k) \Pi_{1} \psi(k)<0$ is equivalent to the existence of matrices $\mathbf{P}>0, \mathbf{Q}>0, \mathbf{R}>0, \mathbf{M}_{0}$, and $\mathbf{M}_{1}$ and a scalar $\lambda \geq 0$ such that

$$
\left[\begin{array}{cccccc}
-\mathbf{M}_{a} & \mathbf{M}_{b}^{T} & 0 & \widetilde{\mathbf{A}}^{T} & \widetilde{\mathbf{A}}_{f}^{T} & \widetilde{\mathbf{M}}_{0}^{T} \\
\mathbf{M}_{b} & -\mathbf{M}_{c} & 0 & \overline{\mathbf{A}}_{d}^{T} & 0 & \mathbf{M}_{1}^{T} \\
0 & 0 & -\lambda \mathbf{I} & \overline{\mathbf{I}} & \mathbf{I} & 0 \\
\widetilde{\mathbf{A}} & \overline{\mathbf{A}}_{d} & \overline{\mathbf{I}}^{T} & -\mathbf{P}^{-1} & 0 & 0 \\
\widetilde{\mathbf{A}}_{f} & 0 & \mathbf{I} & 0 & -\bar{\tau}^{-1} \mathbf{R}^{-1} & 0 \\
\widetilde{\mathbf{M}}_{0} & \mathbf{M}_{1} & 0 & 0 & 0 & -\bar{\tau}^{-1} \mathbf{R}
\end{array}\right]<0 .
$$

Define matrix

$$
\widehat{\mathbf{J}}=\left[\begin{array}{cccccc}
-\mathbf{M}_{a} & \mathbf{M}_{b}^{T} & 0 & \overline{\mathbf{A}}^{T} & \overline{\mathbf{A}}_{f}^{T} & \widetilde{\mathbf{M}}_{0}^{T} \\
\mathbf{M}_{b} & -\mathbf{M}_{c} & 0 & \overline{\mathbf{A}}_{d}^{T} & 0 & \mathbf{M}_{1}^{T} \\
0 & 0 & -\lambda \mathbf{I} & \overline{\mathbf{I}} & \mathbf{I} & 0 \\
\overline{\mathbf{A}} & \overline{\mathbf{A}}_{d} & \overline{\mathbf{I}}^{T} & -\mathbf{P}^{-1} & 0 & 0 \\
\overline{\mathbf{A}}_{f} & 0 & \mathbf{I} & 0 & -\bar{\tau}^{-1} \mathbf{R}^{-1} & 0 \\
\widetilde{\mathbf{M}}_{0} & \mathbf{M}_{1} & 0 & 0 & 0 & -\bar{\tau}^{-1} \mathbf{R}
\end{array}\right],
$$

and then inequality (26) can be rewritten as

$$
\begin{aligned}
\widehat{\mathbf{J}}+ & {\left[\begin{array}{llllll}
0 & 0 & 0 & \overline{\mathbf{D}}^{T} & \mathbf{D}^{T} & 0
\end{array}\right]^{T} \mathbf{F}\left[\begin{array}{llllll}
\overline{\mathbf{E}} & 0 & 0 & 0 & 0 & 0
\end{array}\right] } \\
& +\left[\begin{array}{llllll}
\overline{\mathbf{E}} & 0 & 0 & 0 & 0 & 0
\end{array}\right]^{T} \mathbf{F}^{T}\left[\begin{array}{llllll}
0 & 0 & 0 & \overline{\mathbf{D}}^{T} & \mathbf{D}^{T} & 0
\end{array}\right] \\
& <0 .
\end{aligned}
$$

By Lemma 2, inequality (28) holds for any matrix $\mathbf{F}(k)$ satisfying $\mathbf{F}^{T}(k) \mathbf{F}(k) \leq \mu \mathbf{I}$, if and only if there exists a positive scalar $\varepsilon \geq \sqrt{\mu}$ such that

$$
\begin{aligned}
\widehat{\mathbf{J}} & +\varepsilon\left[\begin{array}{llllll}
0 & 0 & 0 & \overline{\mathbf{D}}^{T} & \mathbf{D}^{T} & 0
\end{array}\right]^{T} \mathbf{F}\left[\begin{array}{llllll}
0 & 0 & 0 & \overline{\mathbf{D}}^{T} & \mathbf{D}^{T} & 0
\end{array}\right] \\
& +\varepsilon\left[\begin{array}{lllllll}
\overline{\mathbf{E}} & 0 & 0 & 0 & 0 & 0
\end{array}\right]^{T}\left[\begin{array}{llllll}
\overline{\mathbf{E}} & 0 & 0 & 0 & 0 & 0
\end{array}\right]<0 .
\end{aligned}
$$

By Schur complement, inequality (29) is equivalent to (10). This completes the proof.

It is noted that in the matrix inequality (10), the controller parameters $\mathbf{A}_{c}, \mathbf{B}_{c}$, and $\mathbf{C}_{c}$ are unknown and occur in nonlinear fashion; therefore, (10) is not an LMI problem. In the sequel, we will use a method of changing variables [21] to obtain an equivalent matrix inequality representation of the nonlinear matrix inequality (10), which enables us to use the CCL technique to design the output feedback controllers.

Now, we present a sufficient condition for the existence of the output feedback delay compensation controller of the form (3) for the NCS (4).
Theorem 4. Consider the NCS (4) and the cost function $J(k)$. Suppose that for some prescribed matrices $\mathbf{S}_{1}>0, \mathbf{S}_{2}>0$; there exist scalars $\gamma(k)>0, \varepsilon \geq \sqrt{\mu}, n \times n$ matrices $\mathbf{X}>0, \mathbf{Y}>0$, $\mathbf{Q}>0$, and $\mathbf{R}>0$, and matrices $\mathbf{M}_{0} \in \mathfrak{R}^{n \times n}, \mathbf{M}_{1} \in \mathfrak{R}^{n \times n}$, $\widehat{\mathbf{A}} \in \mathfrak{R}^{n \times n}, \widehat{\mathbf{B}} \in \mathfrak{R}^{n \times v}$, and $\widehat{\mathbf{C}} \in \mathfrak{R}^{l \times n}$ such that the following optimization problem is feasible at the initial time step $k=0$ :

$$
\begin{array}{ll}
\text { Minimize } & \gamma(k), \\
\text { subject to } & \Lambda_{1} \leq 0, \\
& \Lambda_{2}>0,
\end{array}
$$

and then the networked predictive control derived from the solutions to the above optimization problem robustly asymptotically stabilizes the system (1), and the cost function satisfies the bound

$$
\max _{[\mathbf{A}(k+i), \mathbf{B}(k+i)], i \geq 0} J(k) \leq \gamma(k)
$$

where

$$
\begin{aligned}
& \Lambda_{1}=\left[\begin{array}{ccccc}
-\gamma(k) \mathbf{I} & * & * & * & * \\
\Theta_{1} & \Omega_{1} & * & * & * \\
\Theta_{2} & 0 & \Omega_{2} & * & * \\
\Theta_{3} & 0 & 0 & \Omega_{3} & * \\
\Theta_{4} & 0 & 0 & 0 & \Omega_{4}
\end{array}\right] \\
& \Lambda_{2}=\left[\begin{array}{ccccccccc}
\Xi_{1} & * & * & * & * & * & * & * & * \\
\mathbf{M}_{2} & \mathbf{M}_{c} & * & * & * & * & * & * & * \\
0 & 0 & \lambda \mathbf{I} & * & * & * & * & * & * \\
\Xi_{2} & \Xi_{6} & \Xi_{7} & \Omega_{5} & * & * & * & * & * \\
\Xi_{3} & 0 & \mathbf{I} & 0 & \bar{\tau}^{-1} \mathbf{R}^{-1} & * & * & * & * \\
\mathbf{M}_{3} & \mathbf{M}_{1} & 0 & 0 & 0 & \bar{\tau}^{-1} \mathbf{R} & * & * & * \\
0 & 0 & 0 & \Xi_{8} & \mathbf{D}^{T} & 0 & \varepsilon^{-1} \mathbf{I} & * & * \\
\Xi_{4} & 0 & 0 & 0 & 0 & 0 & 0 & \varepsilon^{-1} \mathbf{I} & * \\
\Xi_{5} & 0 & 0 & 0 & 0 & 0 & 0 & 0 & \Xi_{9}
\end{array}\right], \\
& \Omega_{1}=\left[\begin{array}{cc}
-\mathbf{C Y C}^{T} & * \\
-\mathbf{N}^{T} \mathbf{C}^{T} & -\mathbf{W}
\end{array}\right] \text {, } \\
& \Omega_{2}=\operatorname{diag} \underbrace{\left\{d_{c}^{-1} \mathbf{Q}_{c}, \ldots, d_{c}^{-1} \mathbf{Q}_{c}\right\}}_{\underline{\tau}}, \\
& \Omega_{3}=\operatorname{diag}\left\{d_{1}^{-1} \mathbf{Q}_{c}, \ldots, d_{\bar{\tau}-\underline{\tau}}^{-1} \mathbf{Q}_{c}\right\}, \\
& \Omega_{4}=\operatorname{diag}\left\{\tau_{1}^{-1} \mathbf{R}_{c}, \ldots, \tau_{\bar{\tau}}^{-1} \mathbf{R}_{c}\right\} \text {, } \\
& \Omega_{5}=\left[\begin{array}{cc}
\mathbf{X} & \mathbf{I} \\
\mathbf{I} & \mathbf{Y}
\end{array}\right] \\
& \mathbf{Q}_{c}=\mathbf{C Q}^{-1} \mathbf{C}^{T} \text {, } \\
& d_{i}=d_{c}+i, \quad i=1, \ldots, \bar{\tau}-\underline{\tau},
\end{aligned}
$$




$$
\begin{aligned}
& \tau_{i}=-\bar{\tau}-1+i, \quad i=1, \ldots, \bar{\tau}, \\
& \mathbf{R}_{c}=\mathbf{C R}^{-1} \mathbf{C}^{T}, \\
& \mathbf{Y}_{i}=\mathbf{y}_{k-i+1 \mid k-i+1}-\mathbf{y}_{k-i \mid k-i}, \quad i=1, \ldots, \bar{\tau}, \\
& \Theta_{1}=\left[\begin{array}{ll}
\mathbf{y}_{k \mid k}^{T} & \widehat{\mathbf{x}}_{k \mid k}^{T}
\end{array}\right]^{T}, \\
& \Theta_{2}=\left[\begin{array}{lll}
\mathbf{y}_{k-1 \mid k-1}^{T} & \cdots & \mathbf{y}_{k-\underline{\tau} \mid k-\underline{\tau}}^{T}
\end{array}\right]^{T}, \\
& \Theta_{3}=\left[\begin{array}{lll}
\mathbf{y}_{k-\underline{\tau}-1 \mid k-\underline{\tau}-1}^{T} & \cdots & \mathbf{y}_{k-\bar{\tau} \mid k-\bar{\tau}}^{T}
\end{array}\right]^{T}, \\
& \Theta_{4}=\left[\begin{array}{lll}
\mathbf{Y}_{1}^{T} & \cdots & \mathbf{Y}_{\bar{\tau}}^{T}
\end{array}\right]^{T}, \\
& \Xi_{1}=\left[\begin{array}{cc}
\mathbf{X}-\mathbf{L} & -\mathbf{Y}^{-1}+\mathbf{L} \\
-\mathbf{Y}^{-1}+\mathbf{L} & \mathbf{Y}^{-1}-\mathbf{L}
\end{array}\right], \\
& \Xi_{2}=\left[\begin{array}{cc}
\mathbf{X A} & \mathbf{X A}+\widehat{\mathbf{A}} \\
\mathbf{A} & \mathbf{A}+\mathbf{B} \widehat{\mathbf{C}}
\end{array}\right], \\
& \Xi_{3}=\left[\begin{array}{ll}
\mathbf{A}-\mathbf{I} & \mathbf{A}-\mathbf{I}+\mathbf{B} \widehat{\mathbf{C}}
\end{array}\right], \\
& \Xi_{4}=\left[\begin{array}{ll}
\mathbf{E}_{1} & \mathbf{E}_{1}+\mathbf{E}_{2} \widehat{\mathbf{C}}
\end{array}\right] \text {, } \\
& \Xi_{5}=\left[\begin{array}{cc}
0 & \widehat{\mathbf{C}} \\
0 & \alpha \mathbf{H}_{2} \widehat{\mathbf{C}}
\end{array}\right] \text {, } \\
& \Xi_{6}=\left[\begin{array}{ll}
\mathbf{C}^{T} \widehat{\mathbf{B}}^{T} & 0
\end{array}\right]^{T} \text {, } \\
& \Xi_{7}=\left[\begin{array}{ll}
\mathbf{X} & \mathbf{I}
\end{array}\right]^{T} \text {, } \\
& \Xi_{8}=\left[\begin{array}{ll}
\mathbf{D}^{T} \mathbf{X} & \mathbf{D}^{T}
\end{array}\right], \\
& \Xi_{9}=\operatorname{diag}\left\{\mathbf{S}_{2}^{-1}, \lambda^{-1} \mathbf{I}\right\}, \\
& \mathbf{M}_{2}=\left[\begin{array}{ll}
\mathbf{M}_{1}^{T}-\mathbf{M}_{0} & \mathbf{M}_{1}^{T}-\mathbf{M}_{0}
\end{array}\right], \\
& \mathbf{M}_{3}=\left[\begin{array}{ll}
\mathbf{M}_{0} & \mathbf{M}_{0}
\end{array}\right] \text {, } \\
& \mathbf{L}=-d_{c} \mathbf{Q}+\mathbf{S}_{1}+\mathbf{M}_{0}+\mathbf{M}_{0}^{T}+\lambda \alpha^{2} \mathbf{H}_{1}^{T} \mathbf{H}_{1} .
\end{aligned}
$$

Proof. First, partition $\mathbf{P}$ and its inverse as

$$
\mathbf{P}=\left[\begin{array}{cc}
\mathbf{X} & \mathbf{M} \\
\mathbf{M}^{T} & \mathbf{Z}
\end{array}\right], \quad \mathbf{P}^{-1}=\left[\begin{array}{cc}
\mathbf{Y} & \mathbf{N} \\
\mathbf{N}^{T} & \mathbf{W}
\end{array}\right],
$$

where $\mathbf{X}>0$ and $\mathbf{Y}>0 \in \mathfrak{R}^{\mathbf{n} \times \mathbf{n}}$. Note the identity $\mathbf{P}^{-1} \mathbf{P}=\mathbf{I}$ gives

$$
\mathbf{M N}^{T}=\mathbf{I}-\mathbf{X Y} .
$$

Define

$$
\mathbf{U}_{1}=\left[\begin{array}{cc}
\mathbf{X} & \mathbf{I} \\
\mathbf{M}^{T} & 0
\end{array}\right], \quad \mathbf{U}_{2}=\left[\begin{array}{cc}
\mathbf{I} & \mathbf{I} \\
0 & \mathbf{N}^{T} \mathbf{Y}^{-1}
\end{array}\right] .
$$

Then,

$$
\mathbf{P}^{-1} \mathbf{U}_{1} \operatorname{diag}\left\{\mathbf{I}, \mathbf{Y}^{-1}\right\}=\mathbf{U}_{2}, \quad \mathbf{U}_{1}^{T} \mathbf{P}^{-1} \mathbf{U}_{1}=\Omega_{5} .
$$

Define the new controller variables as

$$
\begin{aligned}
& \widehat{\mathbf{A}}=\widehat{\mathbf{B}} \mathbf{C}+\mathbf{X B} \widehat{\mathbf{C}}+\mathbf{M A}_{c} \mathbf{N}^{T} \mathbf{Y}^{-1}, \\
& \widehat{\mathbf{B}}=\mathbf{M B}_{c}, \quad \widehat{\mathbf{C}}=\mathbf{C}_{c} \mathbf{N}^{T} \mathbf{Y}^{-1} .
\end{aligned}
$$

Therefore, given $\mathbf{X}>0, \mathbf{Y}>0$, and invertible matrices $\mathbf{M}$ and $\mathbf{N}$, the controller matrices $\mathbf{A}_{c}, \mathbf{B}_{c}$, and $\mathbf{C}_{c}$ can be uniquely determined by $\widehat{\mathbf{A}}, \widehat{\mathbf{B}}$, and $\widehat{\mathbf{C}}$.

Pre- and postmultiply (10) by $\operatorname{diag}\left\{\mathbf{U}_{2}^{T}, \mathbf{I}, \mathbf{U}_{1}^{T}, \mathbf{I}, \mathbf{I}, \mathbf{I}, \mathbf{I}, \mathbf{I}\right\}$ and by $\operatorname{diag}\left\{\mathbf{U}_{2}, \mathbf{I}, \mathbf{U}_{1}, \mathbf{I}, \mathbf{I}, \mathbf{I}, \mathbf{I}, \mathbf{I}\right\}$, respectively, set $\mathbf{U}_{2}^{T} \mathbf{M}_{a} \mathbf{U}_{2}=$ $\Xi_{1}, \mathbf{M}_{b}^{T} \mathbf{U}_{2}=\mathbf{M}_{2}, \mathbf{U}_{1}^{T} \overline{\mathbf{A}} \mathbf{U}_{2}=\Xi_{2}, \overline{\mathbf{A}}_{f} \mathbf{U}_{2}=\Xi_{3}, \widetilde{\mathbf{M}}_{0} \mathbf{U}_{2}=\mathbf{M}_{3}$, $\overline{\mathbf{E}} \mathbf{U}_{2}=\Xi_{4}, \mathbf{U}_{1}^{T} \overline{\mathbf{A}}_{d}=\Xi_{6}$, and $\overline{\mathbf{D}}^{T} \mathbf{U}_{1}=\Xi_{8}$, and consider the change of controller variables (39) and Schur complement, and then (32) can be obtained.

By Theorem 3, the original min-max problem (6) can be redefined as the following optimization problem that minimizes an upper bound $\gamma(k)>0$ on the worst value of the original cost function $J(k)$ :

$$
\begin{array}{ll}
\text { Minimize } & \gamma(k) \\
\text { subject to } & \max _{[\mathbf{A}(k+i), \mathbf{B}(k+i)], i \geq 0} J(k) \leq \bar{V}\left(\overline{\mathbf{x}}_{k \mid k}\right) \leq \gamma(k) .
\end{array}
$$

By Schur complement, $\bar{V}\left(\overline{\mathbf{x}}_{k \mid k}\right) \leq \gamma(k)$ is equivalent to the following matrix inequality:

$$
\left[\begin{array}{ccccc}
-\gamma(k) \mathbf{I} & * & * & * & * \\
\overline{\mathbf{x}}_{k \mid k} & -\mathbf{P}^{-1} & * & * & * \\
\widetilde{\Theta}_{2} & 0 & \widetilde{\Omega}_{2} & * & * \\
\widetilde{\Theta}_{3} & 0 & 0 & \widetilde{\Omega}_{3} & * \\
\widetilde{\Theta}_{4} & 0 & 0 & 0 & \widetilde{\Omega}_{4}
\end{array}\right] \leq 0
$$

where

$$
\begin{gathered}
\widetilde{\Theta}_{2}=\left[\mathbf{x}_{k-1 \mid k-1}^{T}, \ldots, \mathbf{x}_{k-\underline{\tau} \mid k-\underline{\tau}}^{T}\right]^{T}, \\
\widetilde{\Theta}_{3}=\left[\mathbf{x}_{k-\underline{\tau}-1 \mid k-\underline{\tau}-1}^{T}, \ldots, \mathbf{x}_{k-\bar{\tau} \mid k-\bar{\tau}]^{T},}^{T}\right. \\
\widetilde{\Theta}_{4}=\left[z_{1}^{T}, \ldots, z_{\bar{\tau}}^{T}\right]^{T}, \\
\mathbf{z}_{i}=\mathbf{x}_{k+1-i \mid k+1-i}-\mathbf{x}_{k-i \mid k-i}, \quad i=1, \ldots, \bar{\tau}, \\
\widetilde{\Omega}_{2}=\operatorname{diag} \underbrace{\left\{d_{c}^{-1} \mathbf{Q}^{-1}, \ldots, d_{c}^{-1} \mathbf{Q}^{-1}\right\}}_{\underline{\tau}}, \\
\widetilde{\Omega}_{3}=\operatorname{diag}\left\{d_{1}^{-1} \mathbf{Q}^{-1}, \ldots, d_{\bar{\tau}-\underline{\tau}}^{-1} \mathbf{Q}^{-1}\right\}, \\
\widetilde{\Omega}_{4}=\operatorname{diag}\left\{\tau_{1}^{-1} \mathbf{R}^{-1}, \ldots, \tau_{\bar{\tau}}^{-1} \mathbf{R}^{-1}\right\} .
\end{gathered}
$$

Define $\mathbf{K}_{2}=\operatorname{diag}\left\{\mathbf{I},\left[\begin{array}{ll}\mathbf{C} & \mathbf{I}\end{array}\right], \mathbf{C}, \ldots, \mathbf{C}\right\}$ and pre- and postmultiply (41) by $\mathbf{K}_{2}$ and $\mathbf{K}_{2}^{T}$, respectively, and it follows from (35) and $\mathbf{y}_{k \mid k}=\mathbf{C x}_{k \mid k}$ that inequality (41) is equivalent to inequality (31). This completes the proof.

Remark 5. Theorem 4 presents an optimization problem to construct the desired output feedback model predictive controllers. Note that the conditions in Theorem 4 are no 
more LMI conditions due to the terms $\mathbf{Y}, \mathbf{Y}^{-1}, \mathbf{Q}, \mathbf{Q}^{-1}, \mathbf{R}$, and $\mathbf{R}^{-1}$. As a result, we cannot find a minimum guaranteed cost by using convex optimization algorithms. However, by using a complementarity idea [18], we can cast the original nonconvex optimization problem to a nonlinear minimization problem involving LMI constraints and, by applying a related iterative algorithm, some suboptimal guaranteed costs can be obtained.

Replace the terms $\mathbf{Y}^{-1}, \mathbf{Q}^{-1}, \mathbf{R}^{-1}$, and $\varepsilon^{-1}$ in $\Lambda_{1}, \Lambda_{2}$ by $\overline{\mathbf{Y}}, \overline{\mathbf{Q}}, \overline{\mathbf{R}}$, and $\bar{\varepsilon}$, respectively, and denote the obtained matrices by $\bar{\Lambda}_{1}, \bar{\Lambda}_{2}$, respectively. Let the cost bound $\gamma(k)$ be lower than some specific value $J^{*}(k)$, and then the nonlinear minimization problem involving LMI constraints can be formulated as follows:

$$
\begin{array}{ll}
\operatorname{minimize} & \operatorname{Trace}(\mathbf{Y} \overline{\mathbf{Y}}+\mathbf{Q} \overline{\mathbf{Q}}+\overline{\mathbf{R}} \mathbf{R}), \\
\text { subject to } & \bar{\Lambda}_{1} \leq 0, \quad \bar{\Lambda}_{2}>0, \quad \gamma(k) \leq J^{*}(k), \\
& {\left[\begin{array}{ll}
\mathbf{Y} & \mathbf{I} \\
\mathbf{I} & \overline{\mathbf{Y}}
\end{array}\right] \geq 0, \quad\left[\begin{array}{cc}
\mathbf{Q} & \mathbf{I} \\
\mathbf{I} & \overline{\mathbf{Q}}
\end{array}\right] \geq 0, \quad\left[\begin{array}{cc}
\overline{\mathbf{R}} & \mathbf{I} \\
\mathbf{I} & \mathbf{R}
\end{array}\right] \geq 0 .}
\end{array}
$$

If the minimum of the above nonlinear minimization problem is 3, that is, Trace $(\mathbf{Y G}+\mathbf{Q U}+\widetilde{\mathbf{R}} \mathbf{R})=3$, we can say from Theorem 4 that the closed-loop system (4) is asymptotically stable with guaranteed cost $\gamma(k)$. We propose an iterative algorithm shown in the following paragraph to solve the above nonlinear minimization problem. Since it is numerically very difficult in practice to obtain the optimal solution such that Trace $(\mathbf{Y G}+\mathbf{Q} \mathbf{U}+\widetilde{\mathbf{R}} \mathbf{R})$ is exactly equal to 3 , we use (31) and (32) as a stopping criterion in the iterative algorithm, and, thus, only some suboptimal guaranteed costs can be obtained within a specified number of iterations.

Now, we summarize the proposed robust networked predictive control algorithm as follows (Figure 1).

(1) Delay Evaluation. Assuming that all clocks of the nodes in the NCS are synchronized and the message is time stamped, the delay $\tau^{s c}$ can be obtained easily. In general, the network-induced delays are time varying, but the approximate probability distributions are similar. Hence, we can evaluate $\tau_{k}^{s a}$ as follows:

$$
\tau_{k}^{s a}=\tau_{k}^{s c}+\tau_{k}^{c a}=t_{k}^{c}-t_{k}^{s}+\frac{1}{\rho} \sum_{l=0}^{\rho-1}\left(t_{k-l}^{c}-t_{k-l}^{s}\right),
$$

where $t_{k}^{c}$ is the time when controller receives the data, $t_{k}^{s}$ is the time when the sensor samples the data, and $\rho$ is the length of the delay window. If the delay $\tau_{k}^{s a}$ is longer than one sampling period $h$, then it can be divided into two parts $\tau_{k}^{s a}=(\tau(k)-$ 1) $h+\widehat{\tau}_{k}$, where $\tau(k)$ is an integer and $0<\widehat{\tau}_{k} \leq h$.

Remark 6. The main objective of the proposed networked predictive control algorithm is to provide the control strategy to compensate the time-varying delays in the case that $\tau_{k}^{s a}$ can be estimated and the effectiveness of the proposed control strategy depends on the exactness of the estimate

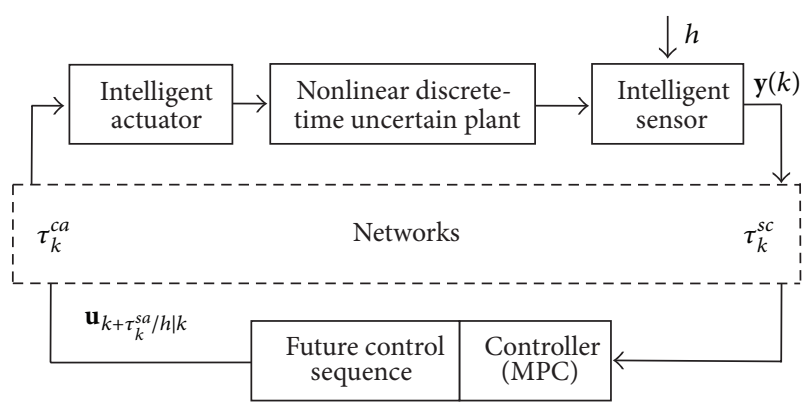

FIGURE 1: A robust networked predictive control system.

for $\tau_{k}^{s a}$; that is, the more exact the estimate for $\tau_{k}^{s a}$ is, the more effective the proposed MPC algorithm will be. In this paper, a commonly used delay evaluation method is adopted just for reference, and, fortunately, a number of advanced delay evaluation methods have now been presented; see, for example, the master-slave clock synchronization technology [22], the Markov or Poisson process based evaluation method [23], and the virtual-queuing-based evaluation method [24]. Some better results can be obtained by applying these newly developed techniques. Using the proposed robust networked predictive control algorithm, the effect of the transmission lag of the manipulated variables can still be eliminated to some extent in the case that $\tau_{k}^{s a}$ cannot be exactly estimated.

(2) The Iterative Algorithm. At time step $k$, the nonlinear minimization problem is solved by using the iterative algorithm shown as follows to get a set of solutions $\widehat{\mathbf{A}}, \widehat{\mathbf{B}}, \widehat{\mathbf{C}}, \mathbf{X}, \mathbf{Y}$, $\mathbf{Q}, \overline{\mathbf{R}}$, and so forth and a suboptimal guaranteed cost $\gamma(k)$ :

(a) choose a sufficiently large initial value of $J^{*}(k)$ such that LMIs in (43) are feasible;

(b) find feasible solutions $\mathbf{X}, \mathbf{Y}, \overline{\mathbf{Y}}, \mathbf{Q}, \overline{\mathbf{Q}}, \mathbf{R}, \overline{\mathbf{R}}, \widehat{\mathbf{A}}, \widehat{\mathbf{B}}, \widehat{\mathbf{C}}, \mathbf{M}_{0}$, $\mathbf{M}_{1}$, and $\bar{\varepsilon}$ to the LMI system in (43). Set $i=0$;

(c) solve the optimization problem for the variables $\mathbf{Y}, \overline{\mathbf{Y}}$, $\mathbf{Q}, \overline{\mathbf{Q}}, \mathbf{R}$, and $\overline{\mathbf{R}}$;

$\operatorname{minimize} \operatorname{Trace}\left(\mathbf{Y}_{\mathbf{i}} \overline{\mathbf{Y}}+\overline{\mathbf{Y}}_{\mathbf{i}} \mathbf{Y}+\mathbf{R}_{\mathbf{i}} \overline{\mathbf{R}}+\overline{\mathbf{R}}_{\mathbf{i}} \mathbf{R}+\mathbf{Q}_{\mathbf{i}} \overline{\mathbf{Q}}+\overline{\mathbf{Q}}_{\mathbf{i}} \mathbf{Q}\right)$

subject to $(43)$;

(d) if conditions (31) and (32) are both satisfied within a specified number of iterations $\beta$, set $J^{*}(k)=J^{*}(k)-$ $\Delta J^{*}(k)$ and return to step (b). Otherwise, if condition (31) or (32) is not satisfied, set $i=i+1, \mathbf{Y}_{i+1}=\mathbf{Y}$, $\overline{\mathbf{Y}}_{i+1}=\overline{\mathbf{Y}}, \mathbf{Q}_{i+1}=\mathbf{Q}, \overline{\mathbf{Q}}_{i+1}=\overline{\mathbf{Q}}, \mathbf{R}_{i+1}=\mathbf{R}$, and $\overline{\mathbf{R}}_{i+1}=\overline{\mathbf{R}}$ and go to step (c). If there is no feasible solution to (31) or (32) when $i$ has increased to $\beta$, then exit.

Remark 7. The CCL procedure is executed at each iterative step, so it will take some time to run the above algorithm. However, If a set of solutions $\widehat{\mathbf{A}}, \widehat{\mathbf{B}}, \widehat{\mathbf{C}}, \mathbf{X}, \mathbf{Y}, \mathbf{Q}$, and $\overline{\mathbf{R}}$ are obtained by using the iterative algorithm at the time step $k$, then, at the next time step $k+1$, we can skip step (b) and use the solutions at the time step $k$ to do step (c) directly. 
By adopting the above procedures, the iterative algorithm with a faster converging rate can be obtained. Besides, the controller computational delays are trivial as compared with the network communication delays. Therefore, the proposed networked predictive control algorithm can be applied in an on-line fashion.

(3) Calculation of the Controller Matrices. Using the solutions obtained in step (2), the controller matrices $\mathbf{A}_{c}, \mathbf{B}_{c}$, and $\mathbf{C}_{c}$ can be uniquely determined as follows:

$$
\begin{gathered}
\mathbf{A}_{c}=\mathbf{M}^{-1}(\widehat{\mathbf{A}}-\mathbf{X A}-\widehat{\mathbf{B}} \mathbf{C}-\mathbf{X B} \widehat{\mathbf{C}}) \mathbf{Y N}^{-T}, \\
\mathbf{B}_{c}=\mathbf{M}^{-1} \widehat{\mathbf{B}}, \quad \mathbf{C}_{c}=\widehat{\mathbf{C}} \mathbf{Y} \mathbf{N}^{-T} \\
\mathbf{Y}=\overline{\mathbf{Y}}^{-1}, \quad \mathbf{M}=(\mathbf{I}-\mathbf{X Y}) \mathbf{N}^{-T}
\end{gathered}
$$

(4) Multistep Prediction and Delay Compensation. The timing diagram of the NCS with network-induced delays is shown in Figure 3; it can be seen from Figure 3 that when the manipulated variable $\mathbf{u}(k)$ arrives at the actuator, the plant output has in fact changed from $\mathbf{y}(k)$ to $\mathbf{y}(k+$ $\left.\tau_{k}^{s a} / h\right)$ during data transmission in network. This situation can be viewed as the logging of the manipulated variable, which degrades the system performance and even causes the instability of the feedback control loop. The transmission lag of manipulated variable can be compensated through the method of multistep prediction. Firstly, since the old data $y(k+i-\tau(k)), i=0, \ldots, \tau(k)$ can be obtained at the instant $k+i$, the predicted value of the state of the output feedback controller $\widehat{\mathbf{x}}_{k+i+1 \mid k}=\mathbf{A}_{c} \widehat{\mathbf{x}}_{k+i \mid k}+\mathbf{B}_{c} \mathbf{y}_{k+i-\tau(k)}, i=0, \ldots, \tau(k)$, and the predicted control inputs $\mathbf{u}_{k+i \mid k}=\mathbf{C}_{c} \widehat{x}_{k+i \mid k}, i=0, \ldots, 1+$ $\tau(k)$, can be calculated based on the MPC strategies. Then, we use the linear interpolation to calculate the predicted control inputs $\mathbf{u}_{k+\tau_{k}^{s a} / h \mid k}$, which is shown in Figure 2. Thus, $\mathbf{u}_{k+\tau_{k}^{s a} / h \mid k}$ can be obtained as follows:

$$
\begin{aligned}
\mathbf{u}_{k+\tau_{k}^{s a} / h \mid k}= & \mathbf{u}_{k+\tau(k)-1 \mid k} \\
& +\left(\mathbf{u}_{k+\tau(k) \mid k}-\mathbf{u}_{k+\tau(k)-1 \mid k}\right) *\left(1+\frac{\tau_{k}^{s a}}{h}-\tau(k)\right) .
\end{aligned}
$$

Therefore, the actual plant input at time step $k$ is $\mathbf{u}(k)=$ $\mathbf{u}_{k+\tau_{k}^{s a} / h \mid k}$.

Remark 8. By multistep prediction, we can obtain the control input $\mathbf{u}_{k+\tau_{k}^{s a} / h \mid k}$, and it can be seen from Figure 3 that when the new manipulated variable $\mathbf{u}_{k+\tau_{k}^{s a} / h \mid k}$ arrives at the actuator, the plant output reaches $\mathbf{y}\left(k+\tau_{k}^{s a} / h\right)$ at the same time, so the effect of the transmission lag of the manipulated variable is eliminated and NCS can be controlled in time and effectively.

Remark 9. By using the multistep prediction and the linear interpolation method, the future control sequence can be obtained. It is assumed that a buffer is introduced at the actuator to store the future control sequence $\Pi_{k}=\left\{u_{k+\tau_{k}^{s a} / h \mid k}\right.$, $\left.u_{k+1+\tau_{k}^{s a} / h \mid k}, \ldots, u_{k+\omega+\tau_{k}^{s a} / h \mid k}\right\}$, where the buffer size $\omega$ is set to be longer than the worst-case delay. It should be noted

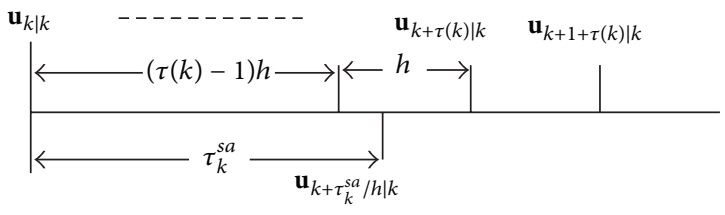

FIgURE 2: The timing diagram for multistep prediction.

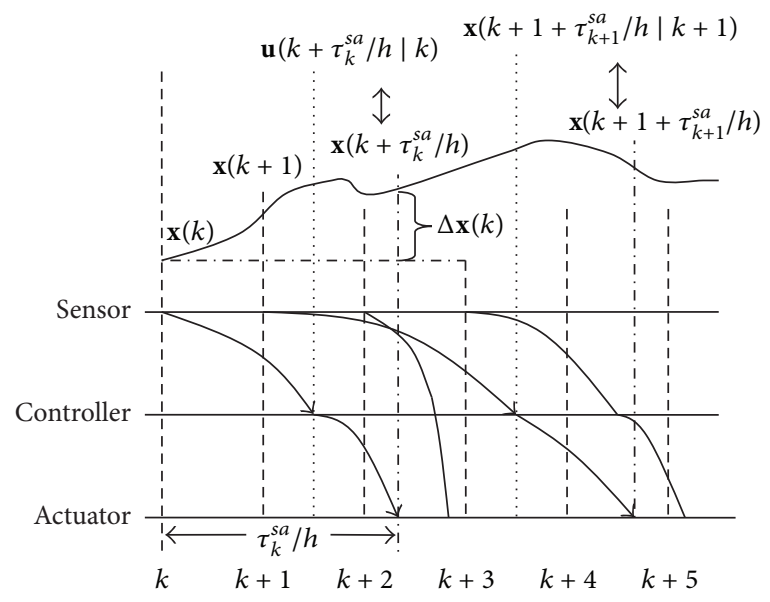

FIGURE 3: The timing diagram for sensors, controllers, and actuators in NCS.

that, with the multistep prediction and the actuator buffering method, we can treat the event of out-of-order data as well as the event of vacant sampling or packet losses. This is because, at a particular time instant, older data that arrive at the controller are used to replace data histories for use in prediction. On the other hand, older data that arrive at the actuator will be discarded if newer data are available. In the case that there is a packet loss, the corresponding control variables in the obtained future control sequence can also be chosen and implemented to eliminate the packet-loss effects. This is true as long as the sequential occurrences of out-oforder data, vacant sampling, or packet losses are within the worst-case delay.

(5) At the next time step, repeat steps (1)-(4) based on the measured output $\mathbf{y}(k)$ and the value $\widehat{\mathbf{x}}(k+1)$ of the controller state.

Remark 10. In the receding horizon framework, only the first computed control move $\mathbf{u}_{k \mid k}$ is implemented. At time step $k+1$, the optimization is solved again with new measurements from the plant. The purpose of taking measurements at each time step is to compensate for the unmeasured disturbances and model uncertainty. This is the main feature of the receding horizon control. Following a similar line as the proof of Lemma 6 in [25], we can conclude that the proposed networked predictive control algorithm is solvable at all time steps $k>0$ if (30) is solvable at time step $k=0$. 


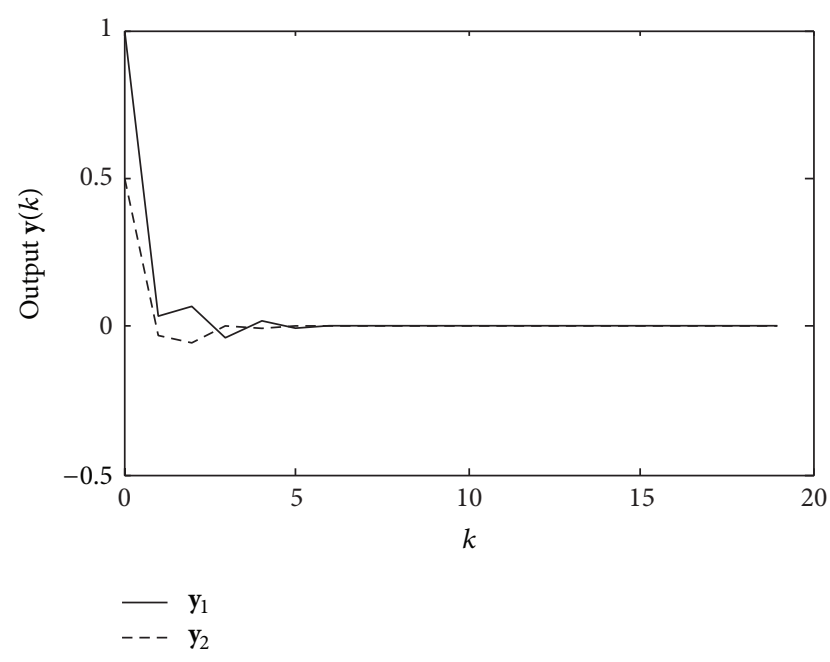

FIgURE 4: Output trajectories.

\section{Illustrative Examples}

In this section, an example is given to illustrate the effectiveness of the proposed robust networked model predictive controllers.

Example 11. Consider the uncertain nonlinear system (1) with the following system matrices:

$$
\begin{gathered}
\mathbf{A}=\left[\begin{array}{cc}
-0.3 & 0.53 \\
-0.28 & 0.4
\end{array}\right], \quad \mathbf{B}=\left[\begin{array}{c}
0.25 \\
1.4
\end{array}\right], \\
\mathbf{C}=\left[\begin{array}{cc}
1 & -1 \\
0 & 0.5
\end{array}\right], \quad \mathbf{D}=\left[\begin{array}{c}
0.2 \\
0.12
\end{array}\right], \\
\mathbf{H}_{1}=\operatorname{diag}\{1,1\}, \quad \mathbf{E}_{1}=\left[\begin{array}{ll}
0.3-0.1
\end{array}\right], \\
\mathbf{E}_{2}=0.7, \quad \mathbf{F}(k)=\sin (k), \\
\mathbf{f}=\mathbf{x}(k) \sin \mathbf{u}(k), \quad \mu=1, \\
\alpha=0.5, \quad \mathbf{H}_{2}=1 .
\end{gathered}
$$

And the initial condition $\widehat{\mathbf{x}}(-i)=\left[\begin{array}{ll}2 & 1\end{array}\right]^{T}, \mathbf{y}(-i)=\left[\begin{array}{ll}1 & 0.5\end{array}\right]^{T}$, for $i=0,1, \ldots, \bar{\tau}$. The sampling time setup is chosen as $h=20 \mathrm{~ms}$. We choose $\boldsymbol{S}_{1}=\operatorname{diag}\{0.5,0.5\}, \boldsymbol{S}_{2}=1$ in the cost function $J(k)$ defined in (4). Simulations are performed for the various values of $\tau(k)$ from 2 to 4 .

We apply the proposed robust output feedback MPC algorithm to stabilize the NCS, and the simulation results are shown in Figures 4-7; Figure 4 shows the output trajectories of the NCS. Figure 5 shows the control signals. The timevarying delays are shown in Figure 6. The values of $\gamma(k)$ at each time step are shown in Figure 7. The figures illustrate that the designed control inputs lead to the stability of the NCS and guarantee an on-line suboptimal receding horizon guaranteed cost at each time step. Therefore, our method is effective.

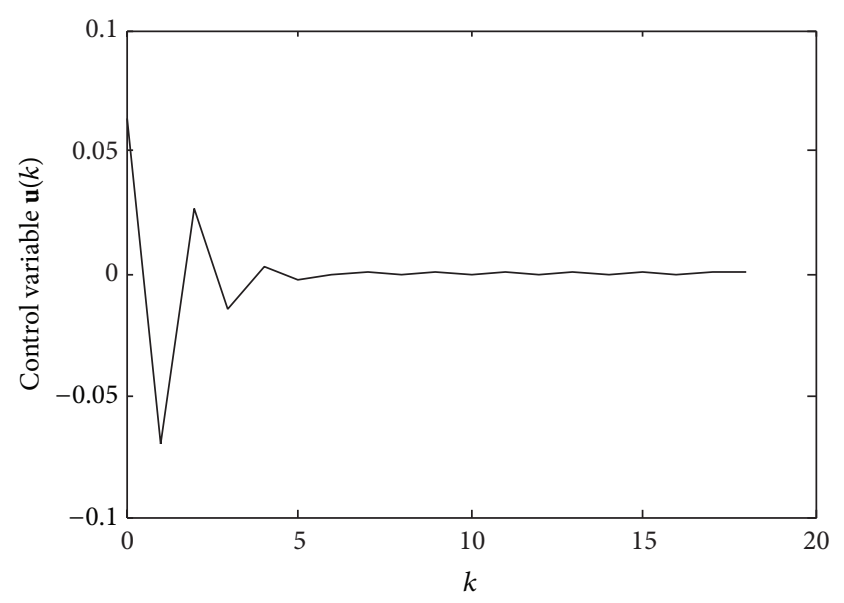

FIGURE 5: Control signals.

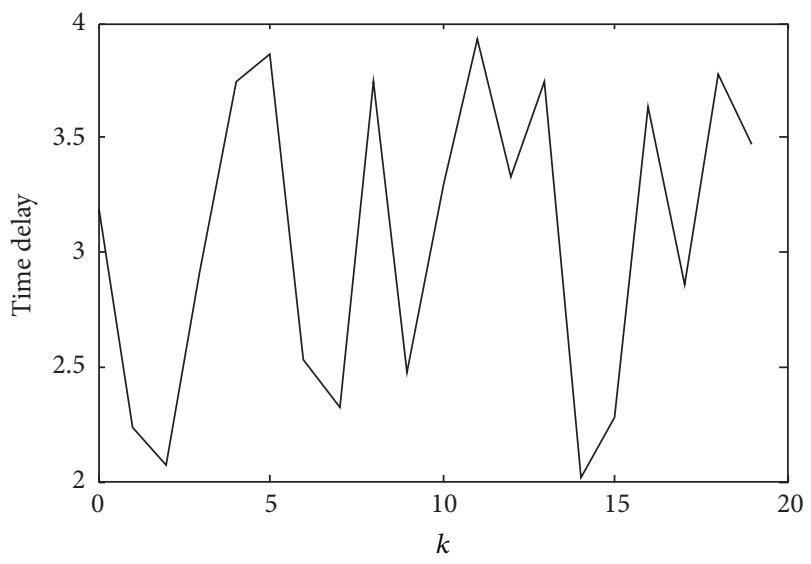

FIgURE 6: Time-varying delays.

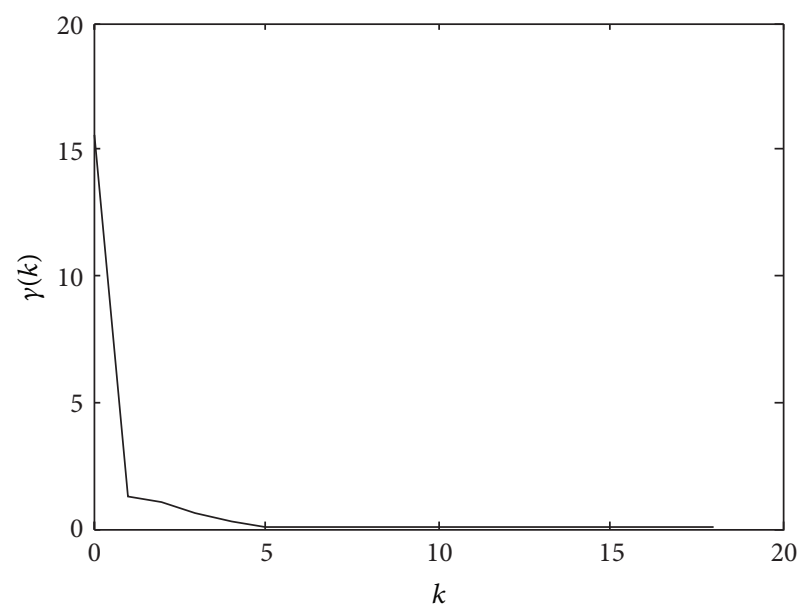

FIGURE 7: Guaranteed costs.

\section{Conclusion}

In this paper, we present a robust output feedback MPC method for the uncertain nonlinear discrete-time systems with time-varying network communication delays. The sideeffect of the transmission lag of the manipulated variables is 
eliminated by using the method of delay evaluation and multistep prediction. By using the MPC strategies and the LMI techniques, a delay-dependent sufficient condition is derived for the existence of the dynamic output feedback controllers, and an on-line optimization algorithm is also presented to construct the desired controllers with a suboptimal guaranteed cost at each time step. Besides, it is not necessary to tune any parameter in the optimization algorithm. Therefore, it is quite suitable for practical applications.

\section{Conflict of Interests}

The authors declare that there is no conflict of interests regarding the publication of this paper.

\section{Acknowledgments}

This work is partially supported by the Environmental Projects of Zhejiang Province under Grant 2013A032, the Public Projects of Zhejiang Province under Grants 2014C33044 and 2012C32021, the Project of Education Department of Zhejiang Province under Grant Y201327680, and Research Center for Smart Agriculture and Forestry under Grant 2013ZHNL02.

\section{References}

[1] A. Bemporad, M. Heemels, and M. Johansson, Networked Control Systems, vol. 406 of Lecture Notes in Control and Information Sciences, Springer, London, UK, 2010.

[2] Y. Sun and N. H. El-Farra, "Quasi-decentralized model-based networked control of process systems," Computers and Chemical Engineering, vol. 32, no. 9, pp. 2016-2029, 2008.

[3] R. A. Gupta and M. Y. Chow, "Networked control system: overview and research trends," IEEE Transactions on Industrial Electronics, vol. 57, no. 7, pp. 2527-2535, 2010.

[4] D. Zhang, Q.-G. Wang, L. Yu, and H. Song, "Fuzzy-model-based fault detection for a class of nonlinear systems with networked measurements," IEEE Transactions on Instrumentation and Measurement, vol. 62, no. 12, pp. 3148-3159, 2013.

[5] D. Zhang, Q.-G. Wang, L. Yu, and Q.-K. Shao, " $H_{\infty}$ filtering for networked systems with multiple time-varying transmissions and random packet dropouts," IEEE Transactions on Industrial Informatics, vol. 9, no. 3, pp. 1705-1716, 2013.

[6] Y. Zhang and H. Fang, "Stabilization of nonlinear networked systems with sensor random packet dropout and time-varying delay," Applied Mathematical Modelling, vol. 35, no. 5, pp. 22532264, 2011.

[7] M. C. F. Donkers, W. P. M. Heemels, D. Bernardini, A. Bemporad, and V. Shneer, "Stability analysis of stochastic networked control systems," Automatica, vol. 48, no. 5, pp. 917-925, 2012.

[8] N. W. Bauer, P. J. H. Maas, and W. P. M. Heemels, "Stability analysis of networked control systems: a sum of squares approach," Automatica, vol. 48, no. 8, pp. 1514-1524, 2012.

[9] W. J. Xu, Z. Zhou, and Q. Liu, "Hybrid one-way delay estimation for networked control system," Advances in Engineering Software, vol. 41, no. 5, pp. 705-711, 2010.

[10] H. Li, H. Yang, F. Sun, and Y. Xia, "A network-bound-dependent stabilization method of networked control systems," Automatica, vol. 49, no. 8, pp. 2561-2566, 2013.
[11] W. A. Zhang and L. Yu, "A robust control approach to stabilization of networked control systems with short time-varying delays," Acta Automatica Sinica, vol. 36, no. 1, pp. 87-91, 2010.

[12] G.-S. Tian, F. Xia, and Y.-C. Tian, "Predictive compensation for variable network delays and packet losses in networked control systems," Computers and Chemical Engineering, vol. 39, pp. 152162, 2012.

[13] Z. H. Pang, G. P. Liu, D. H. Zhou, and M. Y. Chen, "Output tracking control for networked systems: a model-based prediction approach," IEEE Transactions on Industrial Electronics, vol. 61, no. 9, pp. 4867-4877, 2014.

[14] Y. Xia, W. Xie, B. Liu, and X. Wang, "Data-driven predictive control for networked control systems," Information Sciences, vol. 235, pp. 45-54, 2013.

[15] C. Tan and G. Liu, "Consensus of discrete-time linear networked multi-agent systems with communication delays," IEEE Transactions on Automatic Control, vol. 58, no. 11, pp. 29622968, 2013.

[16] G. Pin and T. Parisini, "Networked predictive control of uncertain constrained nonlinear systems: recursive feasibility and input-to-state stability analysis," IEEE Transactions on Automatic Control, vol. 56, no. 1, pp. 72-87, 2011.

[17] D. E. Quevedo and D. Nešić, "Robust stability of packetized predictive control of nonlinear systems with disturbances and Markovian packet losses," Automatica, vol. 48, no. 8, pp. 18031811, 2012.

[18] L. El Ghaoui, F. Oustry, and M. AitRami, "A cone complementarity linearization algorithm for static output-feedback and related problems," IEEE Transactions on Automatic Control, vol. 42, no. 8, pp. 1171-1176, 1997.

[19] D.-S. Kim, Y. S. Lee, W. H. Kwon, and H. S. Park, "Maximum allowable delay bounds of networked control systems," Control Engineering Practice, vol. 11, no. 11, pp. 1301-1313, 2003.

[20] L. Yu and F. Gao, "Optimal guaranteed cost control of discretetime uncertain systems with both state and input delays," Journal of the Franklin Institute, vol. 338, no. 1, pp. 101-110, 2001.

[21] C. Scherer, P. Gahinet, and M. Chilali, "Multiobjective outputfeedback control via LMI optimization," IEEE Transactions on Automatic Control, vol. 42, no. 7, pp. 896-911, 1997.

[22] W. Zhen, M. Xiang-Hua, and X. Jian-Ying, "A novel control scheme based on online delay evaluation for a class of networked control systems," in Proceedings of the American Control Conference (ACC '03), pp. 4143-4148, June 2003.

[23] L. Zhang and F. Hua-Jing, "A novel controller design and evaluation for networked control systems with time-variant delays," Journal of the Franklin Institute, vol. 343, no. 2, pp. 161$167,2006$.

[24] B. Addad, S. Amari, and J.-J. Lesage, "A virtual-queuing-based algorithm for delay evaluation in networked control systems," IEEE Transactions on Industrial Electronics, vol. 58, no. 9, pp. 4471-4479, 2011.

[25] Y. Y. Cao and Z. L. Lin, "Min-max MPC algorithm for LPV systems subject to input saturation," IET Control Theory \& Applications, vol. 152, pp. 266-272, 2005. 


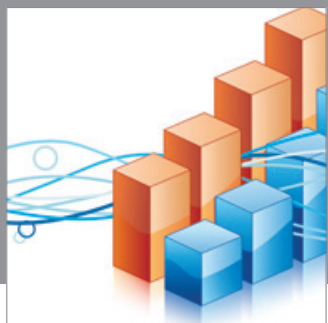

Advances in

Operations Research

mansans

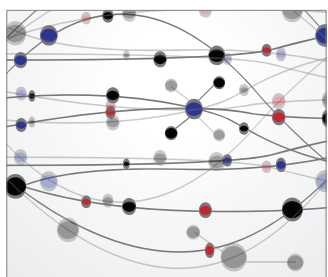

The Scientific World Journal
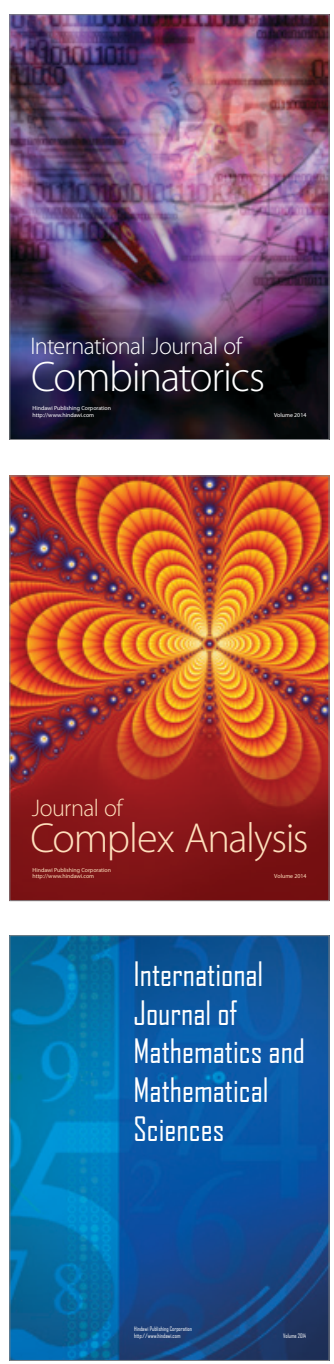
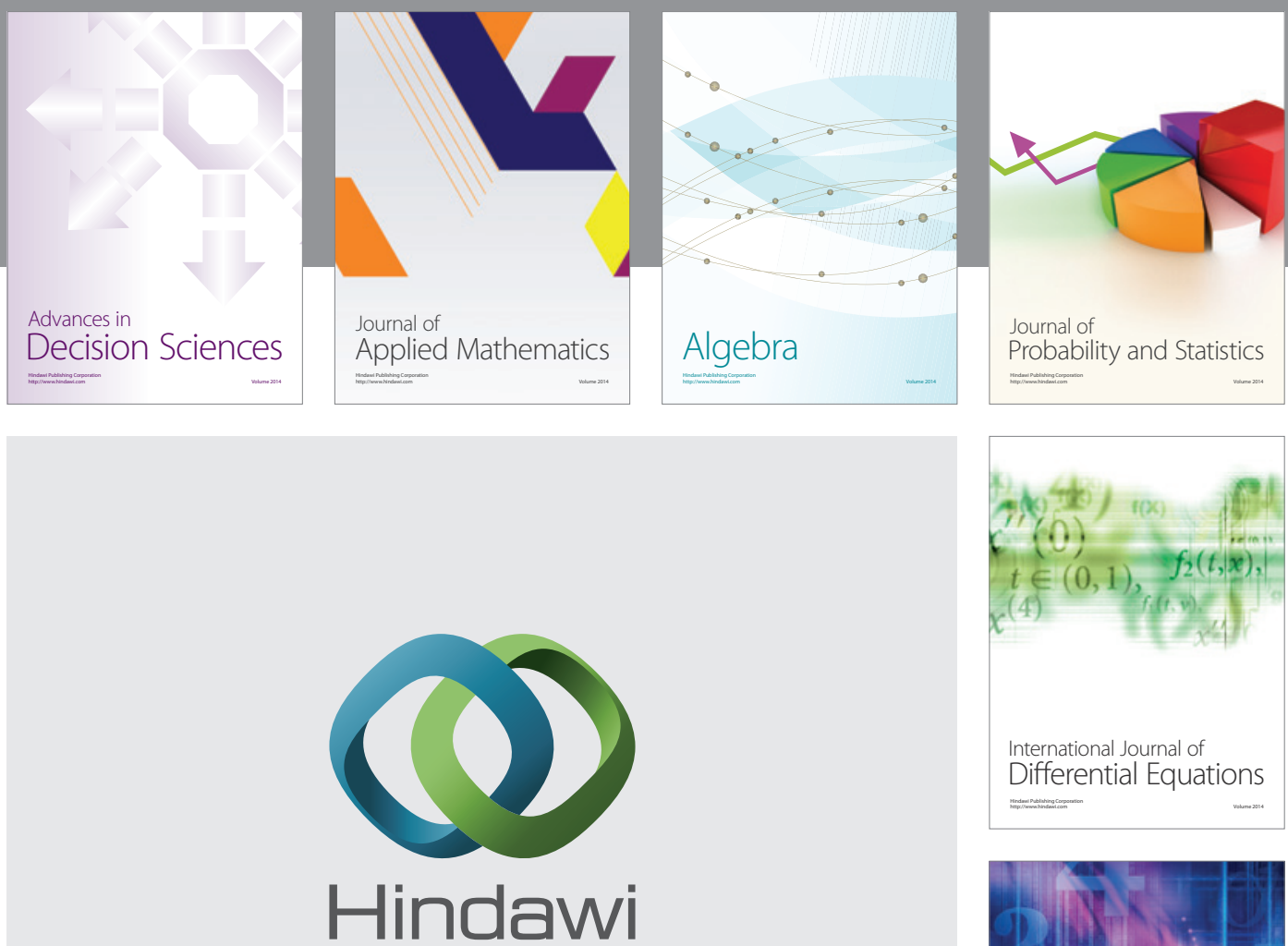

Submit your manuscripts at http://www.hindawi.com
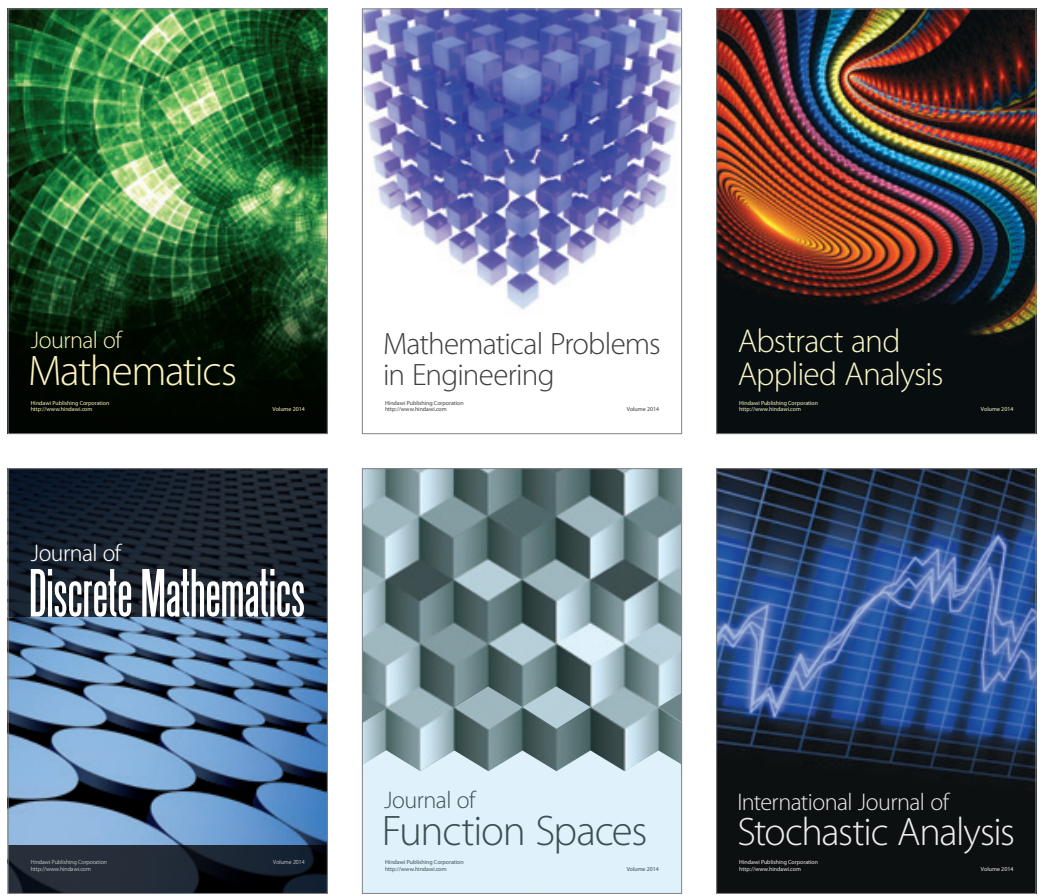

Journal of

Function Spaces

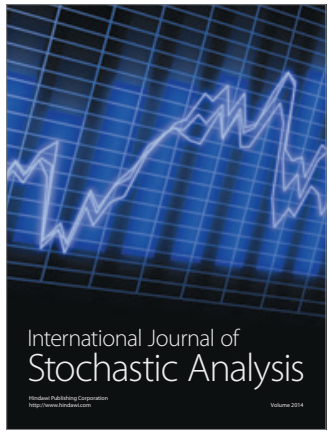

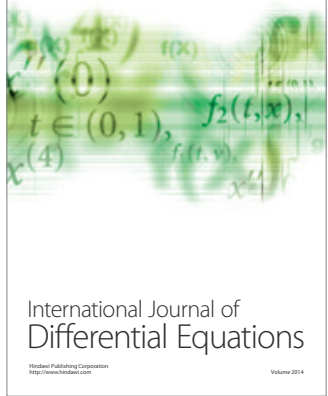
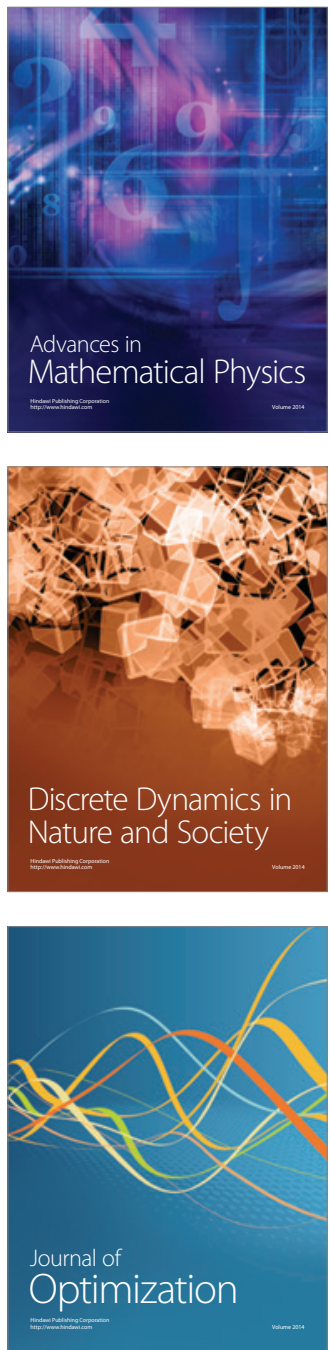